\title{
Helicobacter pullorum sp. nov. - genotype and phenotype of a new species isolated from poultry and from human patients with gastroenteritis
}

\author{
John Stanley, ${ }^{1}$ Dennis Linton, ${ }^{1}$ André P. Burnens, ${ }^{3}$ Floyd E. Dewhirst, ${ }^{4}$ \\ Stephen L. W. On, ${ }^{1}$ Anthony Porter, ${ }^{2}$ Robert J. Owen ${ }^{1}$ and Menelaos \\ Costas $^{1}$
}

Author for correspondence: John Stanley. Tel: +44812004400 ext. 3738. Fax: +44812007874.

\footnotetext{
1,2 National Collection of Type Cultures ${ }^{1}$ and Virus Reference Division². Central Public Health Laboratory, 61 Colindale Avenue, London NW9 5HT, UK

3 Swiss National Reference Laboratory for Foodborne Diseases, Institute for Veterinary Bacteriology, University of Berne, Langass-Strasse $122, \mathrm{CH}$ 3012 Berne, Switzerland

4 Forsyth Dental Center, Department of Molecular Genetics, 140 The Fenway, Boston, MA 02115, USA
}

\begin{abstract}
Campylobacter-like organisms were isolated from the liver, duodenum and caecum of broiler and layer chickens, and from humans with gastroenteritis. They formed a unique DNA homology group and a polyphasic taxonomic analysis was made of 16 strains. Analysis of the nucleotide sequence of the $16 S$ rRNA gene from seven of the strains identified them as belonging to a single species, within the genus Helicobacter. This conclusion was supported by the studies of relative DNA homology and of total protein electrophoretic patterns. The new species could be biochemically differentiated from other helicobacters and its ultrastructure in the electron microscope was typical of the genus except that the flagellum was not sheathed. We propose the name Helicobacter pullorum sp. nov. for this group. Like $H$. fennelliae or $H$. cinaedi it represents another non-gastric urease-negative Helicobacter species colonizing the lower bowel. Its isolation from the livers of chickens with vibrionic hepatitis is significant. We describe a species-specific PCR assay for $H$. pullorum sp. nov. which will facilitate its identification and further studies of its epidemiology.
\end{abstract}

Keywords: Helicobacter pullorum sp. nov., polyphasic taxonomy

\section{INTRODUCTION}

The rRNA homology group VI defined by Vandamme et al. (1991) contains the genera Helicobacter, Campylobacter, Arcobacter and Wolinella in addition to several other poorly defined groups such as 'Flexispira'. The species of greatest current interest are Helicobacter pylori (Blaser, 1993), which is associated with gastroduodenal disease of man (Blaser, 1990), and Campylobacter jejuni, the most common bacterial pathogen causing gastroenteritis in man (Stern, 1993). Helicobacter species are largely associated with specific niches in the gastrointestinal tract of mammalian and avian hosts. For example, $H$. pylori, $H$. mustelae and $H$. felis were isolated from gastric mucosa, and cause gastritis in their respective hosts. Other species (for example, $H$. cinaedi and $H$. fennelliae) can be isolated from human lower-bowel tissue, and are enteric

Abbreviation: CLO, Campylobacter-like organism. pathogens. The genus Helicobacter is currently composed of 11 named species, the most recently defined being $H$. canis (Stanley et al., 1993), H. bepaticus (Fox et al., 1994) and H. pametensis (Dewhirst et al., 1994).

Campylobacter-like organisms (CLOs) were identified in the late 1970 s and early 1980 s as important rectal and intestinal pathogens in homosexual men (Mishu et al., 1993). Two of these three CLOs were subsequently placed in the species Campylobacter cinaedi (CLO-1A) and $C$. fennelliae (CLO-2) (Totten et al., 1985), and ultimately designated Helicobacter cinaedi and Helicobacter fennelliae (Vandamme et al., 1991). Strain CLO-3, of which there was a single example, has remained unnamed (Totten et al., 1985). These studies showed that infectious intestinal pathogens are to be found also in Helicobacter, a genus originally established around the human gastric-adapted bacterium Helicobacter pylori.

This report concerns a group of CLOs which has recently been isolated in Switzerland from three sources: the caeca 
Table 1. Bacterial strains

\begin{tabular}{|c|c|c|c|}
\hline Bacterium & Strain no.* & Source & $\begin{array}{c}\text { GenBank } \\
\text { no. } f\end{array}$ \\
\hline $\begin{array}{l}\text { Helicobacter } \\
\text { H. pylori } \\
\text { H. pylori } \\
\text { H. felis } \\
\text { H. mustelae } \\
\text { H. acinonyx } \\
\text { H. cinaedi } \\
\text { H. fennelliae } \\
\text { H. nemestrinae } \\
\text { H. muridarum } \\
\text { H. canis } \\
\text { H. bepaticus } \ddagger \\
\text { H. pametensis } \ddagger \\
\text { H. sp. Bird-B } \ddagger \\
\text { H. sp. Bird-C } \ddagger \\
\text { 'Chicken CLO' group } \\
\text { (H. pullorum sp. nov.) }\end{array}$ & $\begin{array}{l}\text { NCTC } 11638(\mathrm{R}) \\
\text { NCTC } 11637^{\mathrm{T}} \\
\text { NCTC } 12436^{\mathrm{T}} \\
\text { NCTC } 12435 \\
\text { NCTC } 12686^{\mathrm{T}} \\
\text { NCTC } 12423^{\mathrm{T}} \\
\text { NCTC } 11612^{\mathrm{T}} \\
\text { NCTC } 12491^{\mathrm{T}} \\
\text { NCTC } 12714^{\mathrm{T}} \\
\text { NCTC } 12739^{\mathrm{T}} \\
\text { ATCC } 51448^{\mathrm{T}} \\
\text { CCUG } 29255^{\mathrm{T}} \\
\text { CCUG 29256 (R) } \\
\text { CCUG 29261 (R) } \\
\text { NCTC } 12824^{\mathrm{T}} \\
\text { NCTC } 12825 \\
\text { NCTC } 12826 \\
\text { NCTC } 12827 \\
\text { UB121 } \\
\text { UB122 } \\
\text { UB151 } \\
\text { UB155 } \\
\text { UB1439 } \\
\text { UB4910 } \\
\text { UB4914 } \\
\text { UB5381 } \\
\text { UB3166 } \\
\text { UB3659 } \\
\text { UB4371 } \\
\text { UB4375 }\end{array}$ & $\begin{array}{l}\text { Human } \\
\text { Human } \\
\text { Cat } \\
\text { Ferret } \\
\text { Cheetah } \\
\text { Human } \\
\text { Human } \\
\text { Monkey } \\
\text { Rat } \\
\text { Dog } \\
\text { Mouse } \\
\text { Tern } \\
\text { Tern } \\
\text { Sparrow } \\
\text { Chicken } \\
\text { Chicken } \\
\text { Human } \\
\text { Human } \\
\text { Chicken } \\
\text { Chicken } \\
\text { Chicken } \\
\text { Chicken } \\
\text { Chicken } \\
\text { Chicken } \\
\text { Chicken } \\
\text { Chicken } \\
\text { Human } \\
\text { Human } \\
\text { Chicken } \\
\text { Chicken }\end{array}$ & $\begin{array}{c}\text { M88157 } \\
- \\
\text { M37642 } \\
\text { M35048 } \\
\text { M88148 } \\
\text { M88150 } \\
\text { M88154 } \\
\text { X67854 } \\
\text { M80205 } \\
\text { L13464 } \\
\text { U07574 } \\
\text { M88147 } \\
\text { M88139 } \\
\text { M88144 } \\
\text { L36141 } \\
\text { L36142 } \\
\text { L36143 } \\
\text { L36144 } \\
- \\
- \\
\text { L36145 } \\
- \\
- \\
- \\
- \\
\text { - } \\
\text { L36146 } \\
\text { L36147 } \\
- \\
-\end{array}$ \\
\hline $\begin{array}{l}\text { Campylobacter } \\
\text { C. fetus subsp. fetus } \\
\text { C. coli } \\
\text { C. jejuni subsp. jejuni } \\
\text { C. lari } \\
\text { C. upsaliensis } \\
\text { C. byointestinalis } \\
\text { C. mucosalis } \\
\text { C. sputorum subsp. sputorum } \\
\text { C. concisus } \\
\text { C. curvus } \\
\text { C. rectus } \\
\text { C. belveticus }\end{array}$ & $\begin{array}{l}\text { NCTC } 10842^{\mathrm{T}} \\
\text { NCTC } 11366^{\mathrm{T}} \\
\text { NCTC } 11351^{\mathrm{T}} \\
\text { NCTC } 11352^{\mathrm{T}} \\
\text { NCTC } 11541^{\mathrm{T}} \\
\text { NCTC } 11608^{\mathrm{T}} \\
\text { NCTC } 11000^{\mathrm{T}} \\
\text { NCTC } 11528^{\mathrm{T}} \\
\text { NCTC } 11485^{\mathrm{T}} \\
\text { NCTC } 11649^{\mathrm{T}} \\
\text { NCTC } 11489^{\mathrm{T}} \\
\text { NCTC } 12470^{\mathrm{T}}\end{array}$ & $\begin{array}{l}\text { Sheep } \\
\text { Pig } \\
\text { Cattle } \\
\text { Gull } \\
\text { Dog } \\
\text { Pig } \\
\text { Pig } \\
\text { Human } \\
\text { Human } \\
\text { Human } \\
\text { Human } \\
\text { Cat }\end{array}$ & $\begin{array}{c}\text { L04314 } \\
\text { L04312 } \\
\text { L04315 } \\
\text { L04316 } \\
\text { L14628 } \\
\text { M65010 } \\
\text { L06978 } \\
- \\
\text { L04322 } \\
\text { L04313 } \\
\text { L04317 } \\
\text { U03022 }\end{array}$ \\
\hline $\begin{array}{l}\text { Arcobacter } \\
\text { A. cryaeropbilus } \\
\text { A. butzleri } \\
\text { A. skirrowii } \\
\text { A. nitrofigilis }\end{array}$ & $\begin{array}{l}\text { NCTC } 11885^{\mathrm{T}} \\
\text { NCTC } 12400^{\mathrm{T}} \\
\text { NCTC } 12713^{\mathrm{T}} \\
\text { NCTC } 12251^{\mathrm{T}}\end{array}$ & $\begin{array}{l}\text { Cattle } \\
\text { Human } \\
\text { Sheep } \\
\text { Plant }\end{array}$ & $\begin{array}{l}\text { L14624 } \\
\text { L14626 } \\
\text { L14625 } \\
\text { L14627 }\end{array}$ \\
\hline 'CLO-3' & $\begin{array}{l}\text { NCTC } 12462 \\
(=\text { CCUG } 14564)\end{array}$ & Human & M88151 \\
\hline $\begin{array}{l}\text { 'Flexispira rappini' } \\
\text { Wolinella succinogenes } \\
\text { [Bacteroides] ureolyticus } \$ \\
\text { Salmonella typbimurium }\end{array}$ & $\begin{array}{l}\text { NCTC } 12461^{\mathrm{T}} \\
\text { NCTC } 11488^{\mathrm{T}} \\
\text { NCTC } 10941^{\mathrm{T}} \\
\text { NCTC } 12416^{\mathrm{T}} \text {, } \\
\text { subsp. } 1\end{array}$ & $\begin{array}{l}\text { Human } \\
\text { Cattle } \\
\text { Human } \\
\quad-\end{array}$ & $\begin{array}{c}\text { M88137 } \\
\text { M88159 } \\
\text { L04321 } \\
-\end{array}$ \\
\hline
\end{tabular}

* A superscript $T$ indicates a type strain; $(R)$ indicates a reference strain.

$\dagger 16 \mathrm{~S}$ rRNA sequences for these strains are available for electronic retrieval from GenBank under the indicated accession numbers. The sequences for reference strains deposited in GenBank by F. E. Dewhirst were determined for the strains listed in this Table, but the strains were obtained from different culture collections as indicated by the strain numbers in Fig. 3.

$\ddagger$ Strains included in the $16 \mathrm{~S}$ rRNA gene sequence analysis only.

§Generically misnamed. This species is not a true Bacteroides but belongs in rRNA superfamily VI. 
of asymptomatic poultry (broiler chickens), the livers and intestinal contents of laying hens showing pathological lesions suggestive of vibrionic hepatitis, and finally faecal samples taken from various human patients suffering from gastroenteritis (A. P. Burnens, unpublished results). These isolates could be differentiated from other Campylobacters, and demonstrated to be a homology group by a dot-blot genomic DNA hybridization method (Burnens \& Nicolet, 1992).

We present a polyphasic taxonomic analysis of 16 of these CLO strains (12 from chicken, 4 from humans). We have established the group to be a previously undescribed species of Helicobacter associated with poultry, which is also an infective intestinal pathogen of man. We propose the name Helicobacter pullorum sp. nov. for this new species.

\section{METHODS}

Bacterial strains and culture conditions. Bacterial strains are listed in Table 1. The 16 new CLO strains which are the subject of this study were provisionally referred to as the 'chickenCLO' group, although four isolates were from humans. Helicobacter spp., Campylobacter spp., Arcobacter, Wolinella and Bacteroides spp. were cultured microaerobically or anaerobically as previously described (Stanley et al., 1993).

Phenotypic characterization, protein electrophoresis and electron microscopy. The phenotype of the isolates was determined by employing previously described methods and media (Holmes et al., 1992; On \& Holmes, 1991a, b, 1992). Additional tests were performed as follows. Production of extracellular deoxyribonuclease (DNase) was determined by the method of Lior \& Patel (1987). Growth was tested on nutrient agar (NA) containing $0.05 \%(\mathrm{w} / \mathrm{v})$ safranin $(\mathrm{BDH})$, on NA containing $0.032 \%(\mathrm{w} / \mathrm{v})$ methyl orange $(\mathrm{BDH})$ and on $4.0 \%$ (w/v) $\mathrm{NaCl}$ medium (cf. On \& Holmes, 1991a). The colonial morphology of the isolates was recorded after $3 \mathrm{~d}$ microaerobic incubation on $5 \%(\mathrm{v} / \mathrm{v})$ horse blood agar at $37^{\circ} \mathrm{C}$. Tests were performed in duplicate and with freshly prepared media. Electron microscopy was carried out as previously described (Stanley et al., 1992). Protein samples were prepared, run on SDS-PAGE gels, dried and analysed as described by Costas (1992).

Nucleic acid techniques. Genomic DNA preparation, DNADNA slot-blot hybridization and analysis by laser densitometry were as previously described (Stanley et al., 1992). The parameters governing the DNA homology studies have been discussed previously (Boivin et al., 1985; Lee \& McGee, 1989). Determination of mol\% $\mathrm{G}+\mathrm{C}$ content was by the thermal denaturation method (Owen \& Pitcher, 1985).

Polymerase chain reaction (PCR) amplification of a $1500 \mathrm{bp}$ fragment of the $16 \mathrm{~S}$ rRNA gene of NCTC $12824^{\mathrm{T}}$ and six other strains was done as described previously (Stanley et al., 1993). The amplicon was purified by the method of Kusukawa et al. (1990) and nucleotide sequencing was carried out by the method of Embley (1991). Standard wedge-shaped sequencing gels (Pharmacia LKB Macrophor apparatus) were fixed in aqueous methanol/acetic acid/glycerol $(10 \%, 5 \%, 2 \%, v / v)$, dried at $80^{\circ} \mathrm{C}$ and autoradiographed on Kodak XAR 5 film.

Phylogenetic analysis of 16S rRNA gene sequence and GenBank accession numbers. The $16 \mathrm{~S}$ rRNA gene sequences for NCTC $12824^{\mathrm{T}}$ and six other strains were entered into RNA, a program for analysis of $16 \mathrm{~S}$ rRNA data written in Microsoft
QuickBASIC for use on IBM-PC compatible computers, and aligned as previously described (Paster \& Dewhirst, 1988). The database contains approximately 50 Helicobacter, Wolinella, Arcobacter and Campylobacter sequences and 300 sequences for other eubacteria. Similarity matrices were constructed from aligned sequences by using only those base positions for which $90 \%$ of the strains had data, and were corrected for multiple base changes by the method of Jukes \& Cantor (1969). Phylogenetic trees were constructed by the neighbour-joining method (Saitou \& Nei, 1987). The $16 \mathrm{~S}$ rRNA gene sequences for NCTC $12824^{\mathrm{T}}$, NCTC 12825, NCTC 12826, NCTC 12827 , UB151, UB3166 and UB3659 have been deposited with GenBank; the accession numbers are given in Table 1, which also shows the accession numbers for reference strains included in the phylogenetic analysis.

\section{RESULTS}

\section{Biochemical characterization}

Thirty-two phenotypic tests were performed on the 16 'chicken CLO' strains, resulting in a phenotypic profile which was distinct from the other species within rRNA superfamily VI. All the strains examined produced cytochrome oxidase, reduced nitrate, and grew at both $37^{\circ} \mathrm{C}$ and $42{ }^{\circ} \mathrm{C}$ under microaerobic conditions. All were resistant to cephalothin (blood agar, BA, base, $32 \mathrm{mg}^{-1}$ ), cefoperazone (BA base, $64 \mathrm{mg} \mathrm{l}^{-1}$ ) and methyl orange $(0.32 \%)$. None of the strains produced alkaline phosphatase, DNase or urease. Neither hippurate nor indoxyl acetate was hydrolysed. Growth was not observed at room temperature $\left(18-22^{\circ} \mathrm{C}\right), 25^{\circ} \mathrm{C}$, or $30^{\circ} \mathrm{C}$ under microaerobic conditions, nor aerobically at $37^{\circ} \mathrm{C}$. There was no growth on $0.1 \%$ trimethylamine $N$-oxide (TMAO) medium under anaerobic conditions. Strains were unable to grow on buffered charcoal-yeast or MacConkey media. The strains did not demonstrate tolerance to: $1 \%$ glycine; $2 \%, 3.5 \%$ or $4.0 \% \mathrm{NaCl}$; $0.02 \%$ or $0.05 \%$ safranin; $0.001 \%$ sodium arsenite; or metronidazole ( $4 \mathrm{mg} \mathrm{l}^{-1}$, in BA or NA bases). Most $(14 / 16)$ strains produced catalase, tolerated $1 \%$ bile and were resistant to carbenicillin ( $32 \mathrm{mg} \mathrm{l}^{-\mathbf{1}}$, in BA base). Six strains tolerated $0.1 \%$ potassium permanganate and one was resistant to nalidixic acid $\left(32 \mathrm{mg} \mathrm{ml}^{-1}\right)$. The colonies of all 16 strains on $5 \%$ horse blood agar were pinpointsized, watery, translucent and $\alpha$-haemolytic. No pigments were produced. Table 2 lists phenotypic characteristics useful in distinguishing the new group from other species of Helicobacter.

\section{Whole-cell protein analysis}

One-dimensional SDS-PAGE was carried out in order to analyse whole-cell protein content; about 40 discrete bands were observed. The 16 'chicken CLO' strains were compared with reference or type-strains representing eight Helicobacter species, 'Flexispira rappini', Wolinella succinogenes, and 'CLO-3'. The protein patterns of the new group were homogeneous, other than heterogeneity of several prominent bands in the $39-50 \mathrm{kDa}$ range. After removal of these bands for theoretical reasons previously discussed (Costas, 1992), numerical analysis was performed on the patterns using a correlation coefficient and 
Table 2. Main differential characteristics of $H$. pullorum sp. nov. and related helicobacters

Abbreviations: + , positive; - , negative; $M$, monopolar; B, bipolar; Po, polar; Pe, peritrichous; S, sensitive; (S), most strains sensitive (75-92\%); R, resistant; I, intermediate; ND, not done; NK, not known; V, variable test (35-50\% of strains positive). Source of data: this study and Eaton et al. (1993).

\begin{tabular}{|c|c|c|c|c|c|c|c|c|c|c|c|c|}
\hline Test/characteristic & $\begin{array}{c}\text { H. } \\
\text { pullorum } \\
\text { sp. nov. }\end{array}$ & $\begin{array}{c}H . \\
\text { canis }\end{array}$ & $\begin{array}{c}H . \\
\text { cinaedi }\end{array}$ & $\begin{array}{c}H . \\
\text { fennelliae }\end{array}$ & $\begin{array}{c}H . \\
\text { pylori }\end{array}$ & $\begin{array}{c}H . \\
\text { mustelae }\end{array}$ & $\begin{array}{c}H . \\
\text { muridarum }\end{array}$ & $\begin{array}{c}H . \\
\text { nemestrinae }\end{array}$ & $\begin{array}{c}H . \\
\text { felis }\end{array}$ & $\begin{array}{c}H . \\
\text { acinony } x\end{array}$ & $\begin{array}{c}H . \\
\text { CLO-3 }\end{array}$ & $\begin{array}{c}\text { 'Flexispira } \\
\text { rappini' }\end{array}$ \\
\hline Periplasmic fibres & - & - & - & - & - & - & + & - & + & - & NK & + \\
\hline \multicolumn{13}{|l|}{ Flagella: } \\
\hline Distribution & M & $\mathrm{B}$ & $\mathrm{M}, \mathrm{B}$ & $\mathrm{M}, \mathrm{B}$ & Po & $\mathrm{Pe}$ & B & Po & B & Po & NK & B \\
\hline Number & 1 & 2 & $1-2$ & $1-2$ & $4-8$ & $4-8$ & $10-14$ & $4-8$ & $14-20$ & $2-5$ & $\mathrm{NK}$ & $10-20$ \\
\hline Sheath & - & + & + & + & + & + & + & + & + & + & $\mathrm{NK}$ & + \\
\hline Oxidase production & $16 / 16 t$ & + & + & + & + & + & + & + & + & + & + & + \\
\hline Catalase production & $14 / 16$ & - & + & + & + & + & + & + & + & + & + & + \\
\hline $\begin{array}{l}\text { Urease (rapid) } \\
\text { production }\end{array}$ & $0 / 16$ & - & - & - & + & + & + & + & + & + & - & + \\
\hline Nitrate reduction & $16 / 16$ & - & + & - & - & + & - & - & + & - & - & - \\
\hline $\begin{array}{l}\text { Indoxyl acetate } \\
\text { hydrolysis }\end{array}$ & $0 / 16$ & + & - & + & - & + & + & - & - & - & - & - \\
\hline $\begin{array}{l}\text { Alkaline phosphatase } \\
\text { production }\end{array}$ & $0 / 16$ & + & - & + & + & + & + & + & + & + & + & - \\
\hline \multicolumn{13}{|l|}{ Growth at: } \\
\hline $42{ }^{\circ} \mathrm{C}$ & $16 / 16$ & + & - & - & - & + & - & + & + & - & + & + \\
\hline $25^{\circ} \mathrm{C}$ & $0 / 16$ & - & - & - & - & - & - & - & - & - & - & - \\
\hline \multicolumn{13}{|l|}{ Tolerance to: } \\
\hline $1 \%$ Bile & $13 / 16$ & + & $\mathrm{V}$ & - & - & - & - & - & - & - & - & - \\
\hline Safranin ' $O$ ' & $0 / 16$ & - & - & + & - & - & - & - & - & ND & - & - \\
\hline Methyl orange & $16 / 16$ & - & + & $\mathrm{V}$ & - & - & - & - & - & - & - & - \\
\hline \multicolumn{13}{|l|}{ Sensitivity to: } \\
\hline Nalidixic acid & $15 / 16$ & $\mathrm{~S}$ & S & S & $\mathrm{R}$ & S & $\mathrm{R}$ & $\mathrm{R}$ & $\mathrm{R}$ & $\mathbf{R}$ & $\mathrm{R}$ & $\mathrm{R}$ \\
\hline Cephalothin & $0 / 16$ & $(\mathrm{~S})$ & I & $\mathrm{S}$ & s & $\mathrm{R}$ & $\mathrm{R}$ & S & S & S & $\mathrm{R}$ & $\mathrm{R}$ \\
\hline Cefoperazone & $0 / 16$ & (S) & $(\mathrm{S})$ & $(\mathrm{S})$ & $\mathrm{s}$ & $\mathrm{R}$ & $\mathrm{S}$ & $\mathrm{s}$ & $\mathrm{s}$ & $\mathrm{S}$ & $\mathrm{R}$ & $\mathrm{S}$ \\
\hline $\mathrm{G}+\mathrm{C}$ content $(\mathrm{mol} \%)$ & $34-35$ & 48 & $36-38$ & 35 & $35--37$ & 36 & 34 & 24 & 43 & 30 & 47 & 34 \\
\hline
\end{tabular}

$\dagger$ Number of positive strains/number tested.

UPGMA clustering. The resulting rooted dendrogram is shown in Fig. 1. The 'chicken CLO' strains formed a distinct cluster at the $84 \%$ similarity level. The nearest neighbour to the group was the type strain of $W$. succinogenes, the banding pattern of which was only $55 \%$ similar. The most closely related species of Helicobacter was $H$. felis, the banding pattern of which exhibited $52 \%$ similarity. Campylobacters are not included in the dendrogram (Fig. 1), since they were well separated and since $16 \mathrm{~S}$ rRNA sequence data (see below) indicated that the 'chicken CLOs' were not members of this genus.

\section{Ultrastructure}

Cells were gently curved rods, 3-4 $\mu \mathrm{m}$ in length and $0.3-0.5 \mu \mathrm{m}$ in width. Flagella were single and unipolar; flagellar sheaths were not detected. An electron micrograph of strain NCTC 12827 is shown in Fig. 2.

\section{DNA base composition}

The DNA base compositions of NCTC $12824^{\mathrm{T}}$, NCTC 12826, UB122 and UB3166 were determined as $34 \cdot 5,35 \cdot 1$, 33.8 and $34.8 \mathrm{~mol} \% \mathrm{G}+\mathrm{C}$ respectively.

\section{DNA-DNA hybridization}

The overall genetic relatedness between the chicken CLO strains and the type strains and other reference strains of the species of rRNA superfamily VI (Vandamme et al., 1991) was determined by slot-blot hybridization (Boivin et al., 1985; Lee \& McGee, 1989). When DNA of NCTC $12824^{\mathrm{T}}$ was employed as a probe, the homology was below the limit of detection with DNA of Salmonella typhimurium (negative control), Wolinella succinogenes, Bacteroides ureolyticus, Arcobacter nitrofigilis, Campylobacter belveticus, $C$. upsaliensis, $C$. coli, C. jejuni, C. lari, $C$. byointestinalis, C. fetus, C. mucosalis, C. concisus, $C$. sputorum, C. curvus, C. rectus, 'Flexispira rappini', Helicobacter pylori, $H$. acinonyx, $H$. felis, $H$. fennelliae, $H$. mustelae, $H$. cinaedi, $H$. canis, $H$. nemestrinae or $H$. muridarum.

\section{Sequence of the 165 rRNA gene and phylogenetic analysis of $\boldsymbol{H}$. pullorum sp. nov.}

For NCTC $12824^{\text {T }}$ and the six other 'chicken CLO' strains for which GenBank accession numbers are provided in Table 1 the nucleotide sequence of the 16S rRNA gene was determined as described in Methods. Com- 


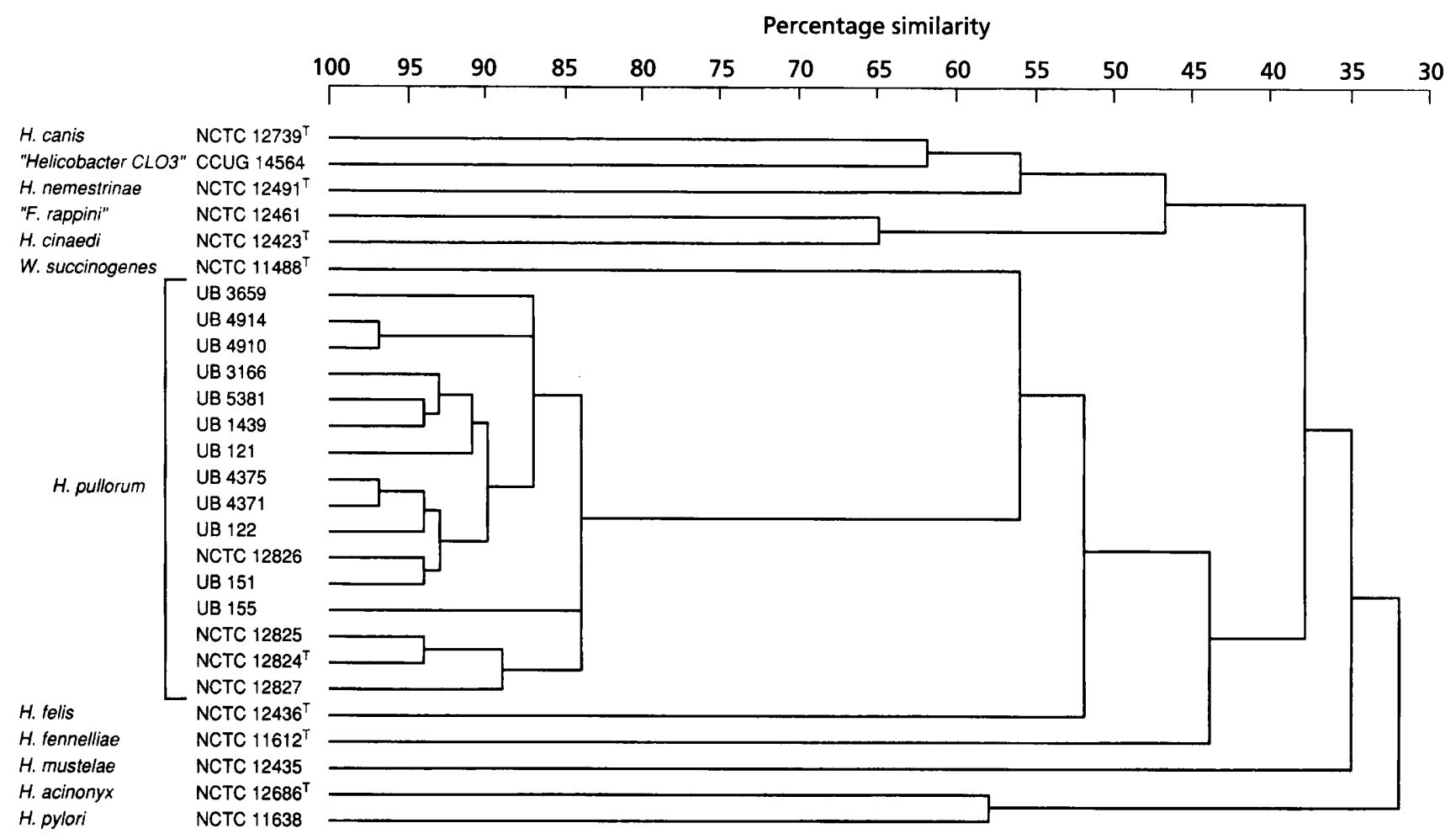

Fig. 1. Dendrogram of numerical analysis of the total protein pattern of 16 'chicken CLO' (H. pullorum sp. nov.) isolates and the type or reference strains of other helicobacters and closely related species.

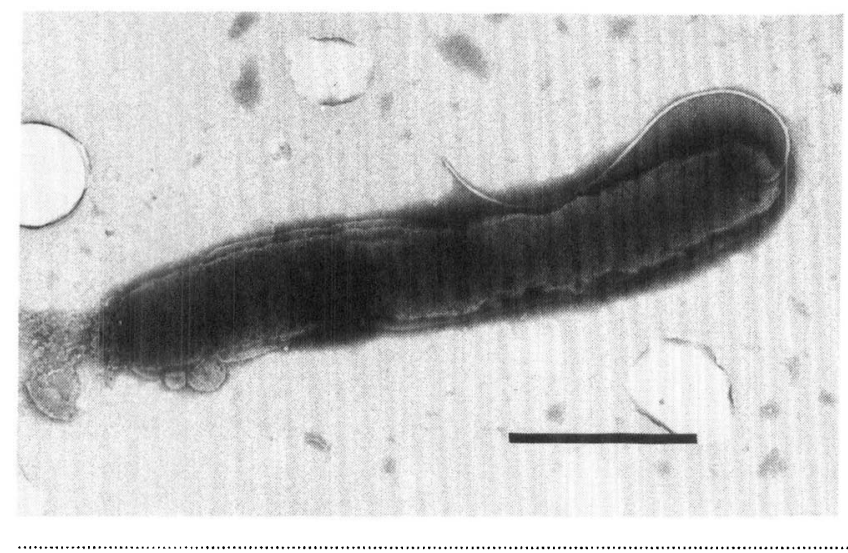

Fig. 2. Electron micrograph of single cell of 'chicken CLO' $(H$. pullorum) NCTC 12827 isolated from an HIV-positive human patient. Note the unipolar flagellum lacking a sheath. Bar, $1 \mu \mathrm{m}$.

parison of these sequences with other eubacterial $16 \mathrm{~S}$ rRNA gene sequences in our database indicated that the strains should be excluded from Campylobacter and placed within the genus Helicobacter. The data for the seven strains were consistent with their belonging to a single species, since only minor sequence variation (1-8 out of 1398 nucleotides) was found in the $16 \mathrm{~S}$ rRNA genes. On this basis we provisionally designated the 'chicken CLO' group as a new species of Helicobacter, $H$. pullorum sp. nov. Results of comparison of the 16S rRNA sequences with those for 27 strains representing Helicobacter, Wolinella,
Arcobacter and Campylobacter species are shown in a matrix (Table 3). Comparisons were made at approximately 1400 base positions, for which data existed for over $90 \%$ of the strains (Table 3 ). The lower half of the matrix is expressed as percentage difference, corrected for multiple base changes by the method of Jukes \& Cantor (1969). NCTC 12824 ${ }^{\mathrm{T}}$ and NCTC 12826 (the most divergent sequences in the new species) were most closely related to another recently described helicobacter, $\mathrm{H}$. pametensis (97.3-97.4\% similarity), to Helicobacter strain CLO-3 (95.7-96.1\% similarity), $H$. canis $(96 \cdot 2-96.8 \%$ similarity), 'Flexispira rappini' (95.9-96.4\% similarity) and $H$. cinaedi $(95 \cdot 5-96 \cdot 1 \%$ similarity). The phylogenetic tree obtained by the neighbour-joining method is shown in Fig. 3. $H$. pullorum sp. nov. formed a distinct phylogenetic lineage within Helicobacter.

\section{A species-specific PCR assay for $H$. pullorum sp. nov.}

The 16S rRNA gene sequences of the seven strains were analysed in order to determine regions which would serve as species-specific priming sites for PCR amplification. A candidate forward primer was identified from nt 818-839, viz. 5' ATG AAT GCT AGT TGT TGT CAG 3'. A candidate reverse primer was identified from nt 12651247, viz. 5' GAT TGG C'TC CAC T'TC ACA 3'. The predicted PCR amplicon was $447 \mathrm{bp}$. Experiments were performed which tested the ability of the primers to amplify such a fragment from the 16 strains of $H$. pullorum sp. nov., and from the type strains of the known species of 
Table 3. Similarity matrix based on 165 rRNA sequence comparisons

Numbers above the diagonal represent uncorrected percentages of similarity, and those below the diagonal are percentages of difference corrected for multiple base changes by the method of Jukes \& Cantor (1969).

\begin{tabular}{|c|c|c|c|c|c|c|c|c|c|c|c|c|c|c|c|c|c|c|c|c|c|c|c|c|c|c|c|c|c|}
\hline & H.pu & H.pu & H.py & H.ne & H.ac & $H_{f} f$ & H.s3 & $H_{f} f n$ & H.mr & H.he & H.ca & H.ci & F.ra & H.pa & H.sC & H.sB & H.ms & W.su & A.cr & A.sk & A.bu & C.re & C.co & C.fe & C.up & C.he & C.la & $C_{; j e}$ & C.co \\
\hline $\begin{array}{l}\text { H. pullorum } \\
12824^{\mathrm{T}}\end{array}$ & - & $99 \cdot 4$ & $94 \cdot 7$ & $94 \cdot 1$ & $94 \cdot 1$ & $94 \cdot 3$ & $96 \cdot 1$ & $95 \cdot 7$ & $95 \cdot 0$ & 95.8 & $96 \cdot 2$ & 95.5 & $95 \cdot 9$ & $97 \cdot 3$ & $96 \cdot 1$ & 95.9 & $95 \cdot 4$ & $92 \cdot 8$ & 86.0 & $85 \cdot 7$ & $86 \cdot 8$ & $85 \cdot 6$ & $87 \cdot 2$ & 86.0 & $87 \cdot 1$ & $86 \cdot 8$ & $87 \cdot 2$ & $86 \cdot 8$ & $86 \cdot 6$ \\
\hline $\begin{array}{l}\text { H. pullorum } \\
12826\end{array}$ & $0 \cdot 6$ & - & $94 \cdot 8$ & $94 \cdot 4$ & $94 \cdot 2$ & $94 \cdot 0$ & $95 \cdot 7$ & $95 \cdot 4$ & $95 \cdot 6$ & $96 \cdot 2$ & 96.8 & $96 \cdot 1$ & $96 \cdot 4$ & $97 \cdot 4$ & $96 \cdot 3$ & $96 \cdot 2$ & $95 \cdot 5$ & $93 \cdot 0$ & $85 \cdot 8$ & $85 \cdot 5$ & $86 \cdot 5$ & $85 \cdot 8$ & $87 \cdot 4$ & $86 \cdot 2$ & $87 \cdot 4$ & $87 \cdot 1$ & $87 \cdot 3$ & $86 \cdot 9$ & $86 \cdot 8$ \\
\hline H. pylori & $5 \cdot 5$ & $5 \cdot 4$ & - & $98 \cdot 2$ & $97 \cdot 4$ & $95 \cdot 4$ & $93 \cdot 8$ & $92 \cdot 9$ & 93.1 & $93 \cdot 3$ & 93.8 & $92 \cdot 7$ & 93.1 & $94 \cdot 4$ & $94 \cdot 1$ & $93 \cdot 7$ & $93 \cdot 8$ & $90 \cdot 8$ & $84 \cdot 6$ & $84 \cdot 2$ & $85 \cdot 4$ & $84 \cdot 0$ & $85 \cdot 0$ & $85 \cdot 0$ & 85.9 & $85 \cdot 4$ & $85 \cdot 4$ & $85 \cdot 3$ & $85 \cdot 1$ \\
\hline H. nemestrinae & 6.2 & 5.9 & 1.8 & - & $96 \cdot 6$ & 95.5 & $93 \cdot 3$ & 92.5 & $92 \cdot 9$ & $93 \cdot 3$ & 93.7 & $93 \cdot 1$ & $93 \cdot 3$ & $94 \cdot 4$ & $94 \cdot 3$ & 93.9 & 93.9 & $90 \cdot 9$ & $84 \cdot 9$ & $84 \cdot 4$ & $85 \cdot 6$ & $84 \cdot 7$ & $85 \cdot 9$ & $85 \cdot 6$ & $86 \cdot 4$ & $85 \cdot 8$ & $85 \cdot 8$ & $85 \cdot 5$ & $85 \cdot 3$ \\
\hline H. acinonyx & $6 \cdot 1$ & 6.0 & $2 \cdot 7$ & 3.5 & - & 96.4 & $93 \cdot 1$ & $92 \cdot 2$ & $92 \cdot 4$ & $93 \cdot 1$ & $93 \cdot 3$ & $92 \cdot 4$ & $92 \cdot 5$ & $94 \cdot 0$ & $93 \cdot 4$ & $93 \cdot 5$ & $93 \cdot 5$ & $90 \cdot 4$ & $84 \cdot 2$ & $84 \cdot 0$ & $85 \cdot 2$ & $83 \cdot 5$ & $84 \cdot 5$ & $84 \cdot 4$ & $85 \cdot 7$ & $85 \cdot 0$ & $85 \cdot 0$ & $84 \cdot 9$ & $84 \cdot 7$ \\
\hline H. felis & 6.0 & 6.2 & $4 \cdot 7$ & $4 \cdot 6$ & $3 \cdot 6$ & - & $93 \cdot 3$ & $93 \cdot 0$ & 92.6 & $93 \cdot 2$ & $93 \cdot 2$ & $92 \cdot 6$ & $92 \cdot 6$ & 93.9 & $93 \cdot 2$ & $93 \cdot 4$ & 93.5 & $90 \cdot 2$ & $83 \cdot 7$ & 83.5 & $84 \cdot 4$ & $83 \cdot 1$ & $84 \cdot 6$ & $84 \cdot 5$ & $86 \cdot 0$ & $85 \cdot 0$ & $85 \cdot 1$ & $84 \cdot 7$ & $84 \cdot 3$ \\
\hline H. sp. CLO-3 & $4 \cdot 0$ & $4 \cdot 4$ & $6 \cdot 4$ & $7 \cdot 0$ & $7 \cdot 3$ & $7 \cdot 1$ & - & $94 \cdot 6$ & $94 \cdot 3$ & $95 \cdot 1$ & $96 \cdot 0$ & $95 \cdot 3$ & $95 \cdot 5$ & $95 \cdot 1$ & $95 \cdot 4$ & $95 \cdot 1$ & $95 \cdot 3$ & $92 \cdot 3$ & $85 \cdot 6$ & $85 \cdot 2$ & $86 \cdot 3$ & $85 \cdot 1$ & $86 \cdot 5$ & $85 \cdot 8$ & 85.9 & $85 \cdot 7$ & $86 \cdot 4$ & 85.8 & $85 \cdot 8$ \\
\hline H. fennelliae & $4 \cdot 4$ & $4 \cdot 8$ & 7.5 & 7.9 & $8 \cdot 3$ & $7 \cdot 3$ & $5 \cdot 6$ & - & $95 \cdot 0$ & $95 \cdot 3$ & $95 \cdot 3$ & $95 \cdot 8$ & $95 \cdot 3$ & 95.4 & $94 \cdot 6$ & $94 \cdot 5$ & $94 \cdot 3$ & $92 \cdot 3$ & $84 \cdot 4$ & $84 \cdot 2$ & $85 \cdot 5$ & $85 \cdot 3$ & $86 \cdot 8$ & $86 \cdot 0$ & $86 \cdot 2$ & $85 \cdot 8$ & 86.6 & $86 \cdot 2$ & $86 \cdot 5$ \\
\hline H. muridarum & $5 \cdot 1$ & 4.5 & $7 \cdot 3$ & $7 \cdot 4$ & $8 \cdot 0$ & $7 \cdot 8$ & 5.9 & $5 \cdot 2$ & - & 97.8 & 96.5 & 95.9 & $96 \cdot 2$ & 95.7 & $96 \cdot 3$ & $96 \cdot 1$ & $95 \cdot 7$ & $93 \cdot 3$ & $85 \cdot 0$ & $84 \cdot 7$ & 85.9 & $84 \cdot 8$ & $86 \cdot 2$ & $85 \cdot 4$ & $86 \cdot 3$ & $86 \cdot 2$ & 86.0 & $85 \cdot 7$ & $85 \cdot 7$ \\
\hline H. bepaticus & $4 \cdot 3$ & 3.9 & $7 \cdot 0$ & $7 \cdot 0$ & $7 \cdot 2$ & $7 \cdot 1$ & $5 \cdot 0$ & $4 \cdot 8$ & $2 \cdot 3$ & - & $97 \cdot 3$ & $96 \cdot 9$ & $97 \cdot 4$ & $96 \cdot 3$ & $96 \cdot 4$ & $96 \cdot 4$ & $96 \cdot 3$ & $92 \cdot 6$ & $84 \cdot 8$ & 84.5 & $85 \cdot 7$ & $85 \cdot 3$ & $86 \cdot 7$ & $85 \cdot 8$ & $86 \cdot 0$ & $86 \cdot 4$ & $86 \cdot 3$ & 85.9 & $86 \cdot 1$ \\
\hline H. canis & 3.9 & $3 \cdot 3$ & 6.5 & 6.6 & $7 \cdot 1$ & $7 \cdot 1$ & $4 \cdot 1$ & $4 \cdot 8$ & $3 \cdot 6$ & $2 \cdot 8$ & -- & $97 \cdot 8$ & $98 \cdot 1$ & 96.5 & $96 \cdot 9$ & $96 \cdot 9$ & $96 \cdot 4$ & $93 \cdot 3$ & $85 \cdot 5$ & $85 \cdot 1$ & $86 \cdot 0$ & $85 \cdot 7$ & $87 \cdot 1$ & $86 \cdot 4$ & 86.9 & $87 \cdot 1$ & $87 \cdot 0$ & $86 \cdot 7$ & 87.0 \\
\hline H. cinaedi & 4.6 & $4 \cdot 0$ & 7.6 & $7 \cdot 2$ & $8 \cdot 0$ & $7 \cdot 8$ & 49 & $4 \cdot 4$ & $4 \cdot 2$ & $3 \cdot 1$ & $2: 2$ & - & $98 \cdot 8$ & 95.5 & $95 \cdot 6$ & $95 \cdot 4$ & $95 \cdot 1$ & $92 \cdot 8$ & $85 \cdot 0$ & $84 \cdot 7$ & $85 \cdot 5$ & $85 \cdot 7$ & $87 \cdot 0$ & $86 \cdot 2$ & $86 \cdot 7$ & $86 \cdot 2$ & $86 \cdot 4$ & $86 \cdot 0$ & $86 \cdot 3$ \\
\hline 'F. rappini' & $4 \cdot 2$ & 3.7 & $7 \cdot 2$ & $7 \cdot 0$ & $7 \cdot 9$ & $7 \cdot 8$ & $4 \cdot 6$ & 4.9 & 3.9 & 2.6 & 20 & $1 \cdot 2$ & - & 96.5 & $96 \cdot 4$ & $95 \cdot 9$ & 95.8 & $93 \cdot 0$ & $85 \cdot 1$ & $84 \cdot 8$ & $85 \cdot 6$ & $85 \cdot 9$ & $87 \cdot 2$ & $86 \cdot 6$ & 86.9 & 87.0 & 87.0 & 86.7 & 87.0 \\
\hline H. pametensis & $2 \cdot 8$ & $2 \cdot 6$ & $5 \cdot 8$ & $5 \cdot 8$ & $6 \cdot 3$ & $6 \cdot 4$ & $5 \cdot 1$ & $4 \cdot 7$ & $4 \cdot 4$ & $3 \cdot 8$ & $2 \cdot 6$ & $4 \cdot 6$ & 3.6 & - & $98 \cdot 1$ & $97 \cdot 9$ & $97 \cdot 1$ & $94 \cdot 1$ & $86 \cdot 1$ & $85 \cdot 7$ & $86 \cdot 5$ & $85 \cdot 9$ & $87 \cdot 4$ & $86 \cdot 4$ & $87 \cdot 3$ & $87 \cdot 1$ & $87 \cdot 5$ & $87 \cdot 2$ & $87 \cdot 0$ \\
\hline H. sp. Bird-C & $4 \cdot 1$ & $3 \cdot 8$ & $6 \cdot 2$ & 5.9 & 6.9 & $7 \cdot 1$ & $4 \cdot 7$ & $5 \cdot 7$ & 3.7 & 3.6 & $3 \cdot 1$ & $4 \cdot 5$ & $3 \cdot 6$ & $2 \cdot 0$ & - & $98 \cdot 3$ & $97 \cdot 9$ & $94 \cdot 5$ & $85 \cdot 6$ & $85 \cdot 2$ & $86 \cdot 3$ & $85 \cdot 5$ & $86 \cdot 6$ & $86 \cdot 4$ & $87 \cdot 4$ & $87 \cdot 2$ & $87 \cdot 5$ & $87 \cdot 1$ & $86 \cdot 7$ \\
\hline H. sp. Bird-B & $4 \cdot 2$ & 3.9 & 6.6 & $6 \cdot 3$ & 6.8 & 6.9 & $5 \cdot 0$ & $5 \cdot 7$ & $4 \cdot 0$ & 3.6 & $3 \cdot 2$ & 4.7 & $4 \cdot 2$ & $2 \cdot 1$ & $1 \cdot 7$ & - & $98 \cdot 6$ & $94 \cdot 1$ & $86 \cdot 2$ & $86 \cdot 0$ & $86 \cdot 9$ & $85 \cdot 1$ & $86 \cdot 4$ & $86 \cdot 1$ & $87 \cdot 0$ & $86 \cdot 8$ & $87 \cdot 1$ & 86.8 & $86 \cdot 5$ \\
\hline H. mustelae & $4 \cdot 8$ & $4 \cdot 6$ & $6 \cdot 4$ & $6 \cdot 3$ & 6.8 & 6.8 & $4 \cdot 9$ & 6.0 & $4 \cdot 4$ & 3.8 & $3 \cdot 7$ & $5 \cdot 0$ & $4 \cdot 3$ & $3 \cdot 0$ & $2 \cdot 1$ & $1 \cdot 4$ & - & $93 \cdot 7$ & $86 \cdot 1$ & $85 \cdot 7$ & $86 \cdot 5$ & $84 \cdot 8$ & $85 \cdot 8$ & $85 \cdot 6$ & $86 \cdot 5$ & $86 \cdot 3$ & $86 \cdot 9$ & $86 \cdot 5$ & 86.2 \\
\hline$W$. succinogenes & $7 \cdot 6$ & $7 \cdot 4$ & $9 \cdot 8$ & $9 \cdot 7$ & $10 \cdot 3$ & $10 \cdot 5$ & $8 \cdot 2$ & $8 \cdot 1$ & $7 \cdot 0$ & $7 \cdot 8$ & $7 \cdot 0$ & 7.5 & $7 \cdot 3$ & $6 \cdot 2$ & $5 \cdot 7$ & 6.2 & 6.6 & - & $85 \cdot 8$ & 85.5 & $86 \cdot 2$ & $86 \cdot 2$ & $86 \cdot 3$ & $85 \cdot 8$ & $86 \cdot 0$ & 85.9 & 86.6 & $86 \cdot 3$ & $86 \cdot 3$ \\
\hline A. cryaerophilus & $15 \cdot 5$ & $15 \cdot 8$ & $17 \cdot 3$ & $16 \cdot 8$ & $17 \cdot 7$ & $18 \cdot 4$ & $16 \cdot 0$ & $17 \cdot 5$ & $16 \cdot 7$ & $17 \cdot 0$ & $16 \cdot 1$ & $16 \cdot 7$ & $16 \cdot 6$ & $15 \cdot 4$ & $16 \cdot 0$ & $15 \cdot 3$ & $15 \cdot 4$ & $15 \cdot 8$ & - & 98.9 & 97.5 & $86 \cdot 0$ & $87 \cdot 5$ & $86 \cdot 2$ & $86 \cdot 0$ & $86 \cdot 4$ & $87 \cdot 1$ & $87 \cdot 4$ & $86 \cdot 8$ \\
\hline A. skirrowii & $15 \cdot 9$ & $16 \cdot 2$ & $17 \cdot 7$ & $17 \cdot 5$ & $18 \cdot 0$ & $18 \cdot 7$ & $16 \cdot 5$ & $17 \cdot 7$ & $17 \cdot 1$ & $17 \cdot 4$ & $16 \cdot 6$ & $17 \cdot 2$ & $17 \cdot 0$ & $15 \cdot 9$ & $16 \cdot 5$ & $15 \cdot 5$ & $15 \cdot 9$ & $16 \cdot 1$ & $1 \cdot 1$ & - & 97.2 & $85 \cdot 6$ & $87 \cdot 6$ & $86 \cdot 1$ & $85 \cdot 9$ & $86 \cdot 1$ & $86 \cdot 8$ & $87 \cdot 2$ & 86.7 \\
\hline A. butqleri & $14 \cdot 6$ & 14.8 & $16 \cdot 2$ & $16 \cdot 0$ & 16.5 & $17 \cdot 5$ & $15 \cdot 1$ & $16 \cdot 1$ & $15 \cdot 7$ & $15 \cdot 9$ & $15 \cdot 6$ & $16 \cdot 2$ & $16 \cdot 0$ & 14.9 & $15 \cdot 1$ & $14 \cdot 4$ & $14 \cdot 9$ & $15 \cdot 2$ & $2 \cdot 6$ & 2.8 & - & $86 \cdot 5$ & $87 \cdot 9$ & $86 \cdot 3$ & $86 \cdot 4$ & $86 \cdot 3$ & $86 \cdot 6$ & 86.9 & 86.4 \\
\hline C. rectus & 16.0 & $15 \cdot 7$ & 18.0 & $17 \cdot 1$ & $18 \cdot 6$ & $19 \cdot 2$ & 16.6 & $16 \cdot 4$ & $17 \cdot 0$ & $16 \cdot 4$ & $15 \cdot 9$ & $15 \cdot 8$ & $15 \cdot 6$ & $15 \cdot 6$ & $16 \cdot 1$ & $16 \cdot 6$ & $17 \cdot 0$ & $15 \cdot 3$ & 15.5 & $16 \cdot 0$ & $14 \cdot 9$ & - & $95 \cdot 9$ & $93 \cdot 8$ & $91 \cdot 7$ & 91.6 & $92 \cdot 4$ & $92 \cdot 7$ & $93 \cdot 4$ \\
\hline C. concisus & $14 \cdot 1$ & $13 \cdot 8$ & 16.8 & $15 \cdot 6$ & $17 \cdot 3$ & $17 \cdot 3$ & 14.9 & 14.5 & $15 \cdot 3$ & $14 \cdot 6$ & $14 \cdot 1$ & $14 \cdot 3$ & $14 \cdot 0$ & 13.8 & $14 \cdot 7$ & $15 \cdot 0$ & $15 \cdot 7$ & $15 \cdot 2$ & 13.6 & 13.5 & $13 \cdot 1$ & $4 \cdot 2$ & - & $95 \cdot 4$ & $92 \cdot 2$ & $92 \cdot 0$ & 93.8 & 93.5 & $94 \cdot 2$ \\
\hline C. fetus & $15 \cdot 5$ & $15 \cdot 3$ & $16 \cdot 7$ & $16 \cdot 0$ & $17 \cdot 5$ & $17 \cdot 3$ & $15 \cdot 8$ & $15 \cdot 4$ & $16 \cdot 2$ & $15 \cdot 7$ & $15 \cdot 0$ & $15 \cdot 2$ & $14 \cdot 8$ & $15 \cdot 1$ & $15 \cdot 0$ & $15 \cdot 4$ & 16.0 & $15 \cdot 7$ & $15 \cdot 3$ & $15 \cdot 4$ & $15 \cdot 2$ & 6.5 & $4 \cdot 8$ & - & $93 \cdot 1$ & $93 \cdot 1$ & $94 \cdot 0$ & $94 \cdot 3$ & $95 \cdot 3$ \\
\hline C. upsaliensis & $14 \cdot 2$ & $13 \cdot 8$ & $15 \cdot 6$ & 14.9 & 15.9 & $15 \cdot 5$ & $15 \cdot 6$ & $15 \cdot 3$ & $15 \cdot 2$ & $15 \cdot 5$ & $14 \cdot 5$ & $14 \cdot 7$ & $14 \cdot 4$ & 13.9 & $13 \cdot 8$ & $14 \cdot 3$ & $14 \cdot 9$ & $15 \cdot 5$ & $15 \cdot 4$ & $15 \cdot 7$ & $15 \cdot 0$ & $8 \cdot 8$ & $8 \cdot 2$ & $7 \cdot 3$ & - & $98 \cdot 2$ & 95.5 & $96 \cdot 1$ & $95 \cdot 1$ \\
\hline C. belveticus & $14 \cdot 5$ & $14 \cdot 2$ & $16 \cdot 3$ & $15 \cdot 7$ & $16 \cdot 8$ & $16 \cdot 8$ & $15 \cdot 9$ & $15 \cdot 7$ & $15 \cdot 2$ & $15 \cdot 0$ & $14 \cdot 1$ & $15 \cdot 2$ & $14 \cdot 2$ & $14 \cdot 1$ & $14 \cdot 0$ & $14 \cdot 6$ & $15 \cdot 1$ & $15 \cdot 6$ & $15 \cdot 0$ & $15 \cdot 4$ & $15 \cdot 2$ & 8.9 & $8 \cdot 4$ & $7 \cdot 3$ & $1 \cdot 8$ & - & $96 \cdot 6$ & 97.2 & $96 \cdot 2$ \\
\hline C. lari & $14 \cdot 1$ & 13.9 & $16 \cdot 2$ & $15 \cdot 7$ & $16 \cdot 8$ & 16.6 & $15 \cdot 0$ & 14.8 & $15 \cdot 5$ & $15 \cdot 1$ & $14 \cdot 2$ & $15 \cdot 1$ & $14 \cdot 2$ & 13.6 & $13 \cdot 7$ & $14 \cdot 1$ & $14 \cdot 4$ & $14 \cdot 7$ & $14 \cdot 2$ & 14.5 & 14.8 & 8.0 & 6.5 & $6 \cdot 3$ & $4 \cdot 6$ & $3 \cdot 5$ & - & 98.6 & $97 \cdot 6$ \\
\hline C. jejuni & $14 \cdot 5$ & $14 \cdot 4$ & $16 \cdot 3$ & $16 \cdot 1$ & $16 \cdot 8$ & $17 \cdot 1$ & $15 \cdot 8$ & $15 \cdot 2$ & 15.8 & $15 \cdot 6$ & $14 \cdot 7$ & $15 \cdot 5$ & $14 \cdot 7$ & $14 \cdot 1$ & $14 \cdot 1$ & $14 \cdot 5$ & $14 \cdot 9$ & $15 \cdot 1$ & 13.8 & $14 \cdot 0$ & $14 \cdot 4$ & $7 \cdot 7$ & 6.8 & 60 & $4 \cdot 0$ & $2 \cdot 9$ & 1.4 & - & $98 \cdot 6$ \\
\hline C. coli & 14.7 & $14 \cdot 6$ & $16 \cdot 6$ & $16 \cdot 4$ & $17 \cdot 1$ & $17 \cdot 7$ & $15 \cdot 8$ & 14.9 & $15 \cdot 8$ & $15 \cdot 3$ & $14 \cdot 3$ & $15 \cdot 1$ & $14 \cdot 3$ & $14 \cdot 3$ & $14 \cdot 7$ & $14 \cdot 9$ & $15 \cdot 2$ & $15 \cdot 2$ & 14.5 & 14.7 & $15 \cdot 0$ & 6.9 & $6 \cdot 1$ & $4 \cdot 8$ & $5 \cdot 1$ & 3.9 & $2 \cdot 5$ & 1.4 & - \\
\hline
\end{tabular}

Campylobacter and Helicobacter, 'Flexispira rappini' and Wolinella succinogenes. Using an annealing temperature of $60^{\circ} \mathrm{C}$, no product was observed from any strain except those belonging to $H$. pullorum sp. nov. From all the latter strains a PCR amplicon of $\sim 450$ bp was detected (Fig. 4).

The microaerophilic nature, cytochrome oxidase activity and general morphology of the 'chicken CLO' strains are typical of a species belonging to rRNA superfamily VI (Vandamme et al., 1991). From the molecular systematic analysis of the group it was evident that it was a previously undescribed Helicobacter species, for which we propose the name $H$. pullorum sp. nov., to indicate its isolation from chickens. This new species is clearly distinguishable biochemically from other species of Helicobacter (see Table 2 ). It can readily be classified into the urease-negative enteric group of Helicobacter species ( $H$. cinaedi, $H$. fennelliae and $H$. canis). The urease enzyme is considered necessary for survival in the acidic environment of the mammalian stomach, and is characteristic of the gastric helicobacters such as $H$. pylori. Its absence in non-gastric Helicobacter species might be explicable on the basis that the deamination of urea in non-acidic environments would yield toxic end-products. Among the enteric helicobacters $H$. pullorum can be distinguished from $H$. fennelliae and $H$. canis by its ability to reduce nitrate and inability to hydrolyse indoxyl acetate. It differs from the Helicobacter strain CLO3 by nitrate reduction, and its inability to produce alkaline phosphatase. The phenotype of $H$. pullorum sp. nov. closely resembles that of $H$. cinaedi, but can be distinguished by growth at $42{ }^{\circ} \mathrm{C}$. It is differentiated biochemically from the recently described avian species $H$. pametensis (to which it is most closely related in terms of $16 \mathrm{~S}$ rRNA gene sequence) by inability to produce alkaline phosphatase, and resistance to cephalothin. The absence of alkaline phosphatase production also distinguishes $H$. pullorum from the other, as yet unnamed strains designated Helicobacter sp. Bird-B and Bird-C in our phylogenetic database (Seymour et al., 1994).

The cellular ultrastructure (Fig. 2) was consistent with assignment to Helicobacter, but the flagellum was found not to be sheathed, the first such case for a Helicobacter species (Vandamme et al., 1991). The monopolar flagellum, cellular ultrastructure and lack of complex surface features resemble those found in $H$. cinaedi and $H$. fennelliae (Han et al., 1989). Indeed, one or two polar flagella seem to be a common feature of the enteric, as opposed to gastric, species of Helicobacter. The 16 strains formed a tight cluster in the rooted dendrogram derived from SDS-PAGE protein analysis (Fig. 1). This cluster was clearly separated from other Helicobacter species, the nearest of which was $H$. felis. The biochemically similar species $H$. cinaedi had a quite distinct total protein pattern 
(\% Difference)

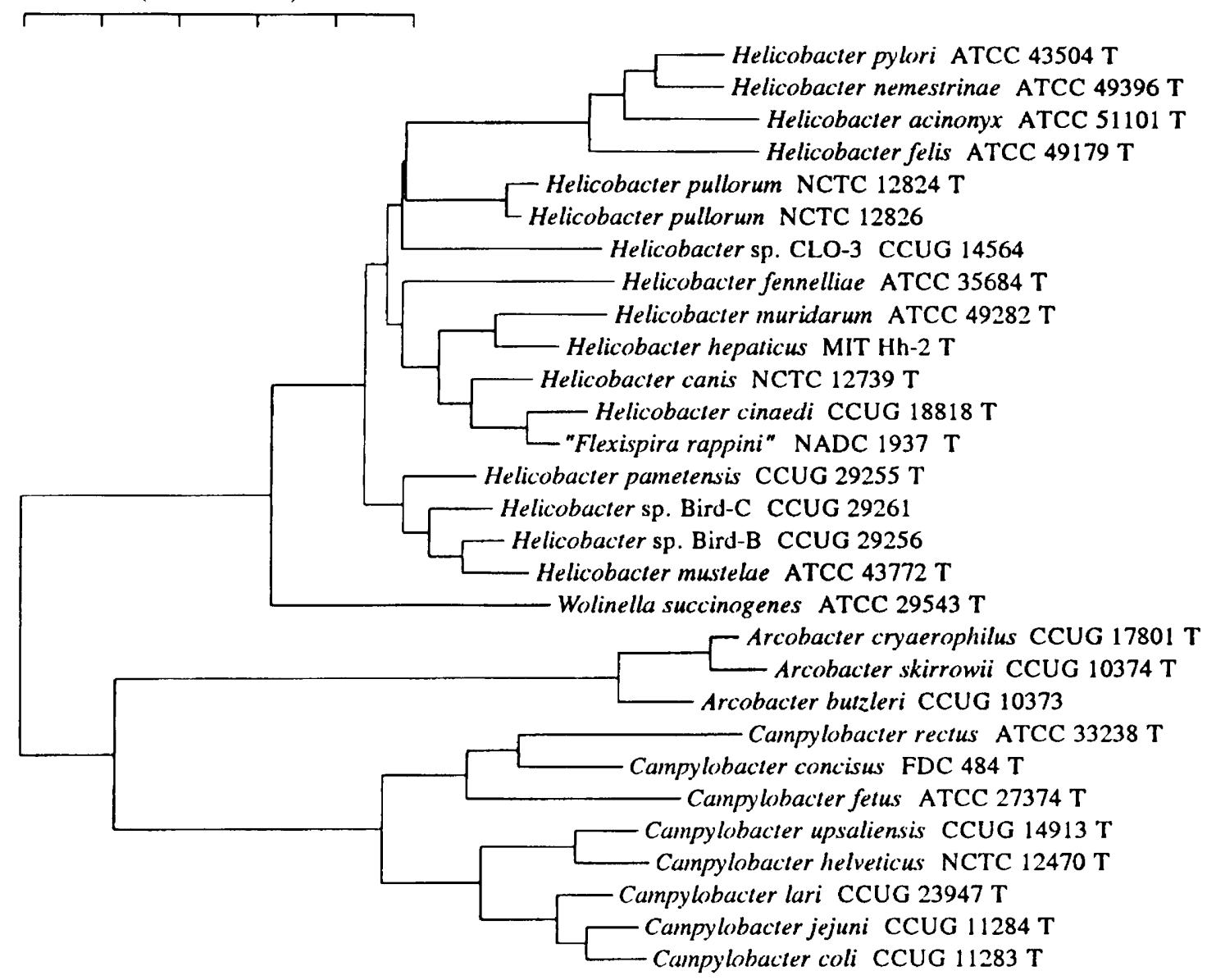

Fig. 3. Phylogenetic tree for two $H$. pullorum strains and 27 reference Helicobacter, Campylobacter, Wolinella and Arcobacter species, based on $16 \mathrm{~S}$ rRNA gene sequence similarity. The scale bar represents $5 \%$ difference in nucleotide sequence as determined by measuring the lengths of the horizontal lines connecting any two species.

(only $45 \%$ similarity) whilst $H$. fennelliae and $H$. canis, other enteric helicobacters, were even less similar (both $\sim 38 \%$ similarity).

Between the seven strains of $H$. pullorum sp. nov. whose $16 \mathrm{~S}$ rRNA genes were sequenced, there was much less than $1 \%$ sequence divergence, consistent with membership of a single species. On the basis of these seven sequences, primers for a species-specific PCR assay could be designed to avoid regions of intraspecific variation. The resulting assay will facilitate definitive identification of the new species.

From the phylogenetic analysis the nearest neighbour of $H$. pullorum sp. nov. was $H$. pametensis, a urease-negative helicobacter isolated from avian and porcine faeces (Dewhirst et al., 1994). H. canis and CLO-3 were related at about the $96 \%$ level. The most related urease-positive gastric species was 'Flexispira rappini'. When the overall genetic relatedness of $H$. pullorum to the other Helicobacter species was assessed by DNA-DNA hybridization, no significant hybridization was observed. This concurs with the electrophoretic analysis of proteins, and the $16 \mathrm{~S}$ rRNA gene phylogeny, in defining a new species of
Helicobacter. The mol \% G+C was also typical of the genus Helicobacter.

It should be noted that $H$. pullorum, like $H$. hepaticus (Fox et al., 1994) and 'Flexispira rappini', resides in the intestinal tract, but has the ability to invade the liver and cause hepatitis in some hosts. This aspect of the organism deserves further study. One-quarter of the strains of $H$. pullorum in this study were isolated from faecal specimens of human patients with diarrhoeal disease; one of these was an immunocompromised individual with human immunodeficiency virus (HIV) infection. These strains clustered with the chicken strains according to all criteria. $H$. pullorum is therefore likely to be an infective enteric pathogen. Its potential as a causative agent of serious gastrointestinal disease in humans is evidenced by the case of strain NCTC 12826, which was isolated from a young woman with chronic diarrhoea of one month's duration. Since the specific route of infection was not established for these human cases, the potential of $H$. pullorum in zoonotic infection should also be made a subject of further study. The PCR identification assay described in this report can be carried out from DNA or from cells and 

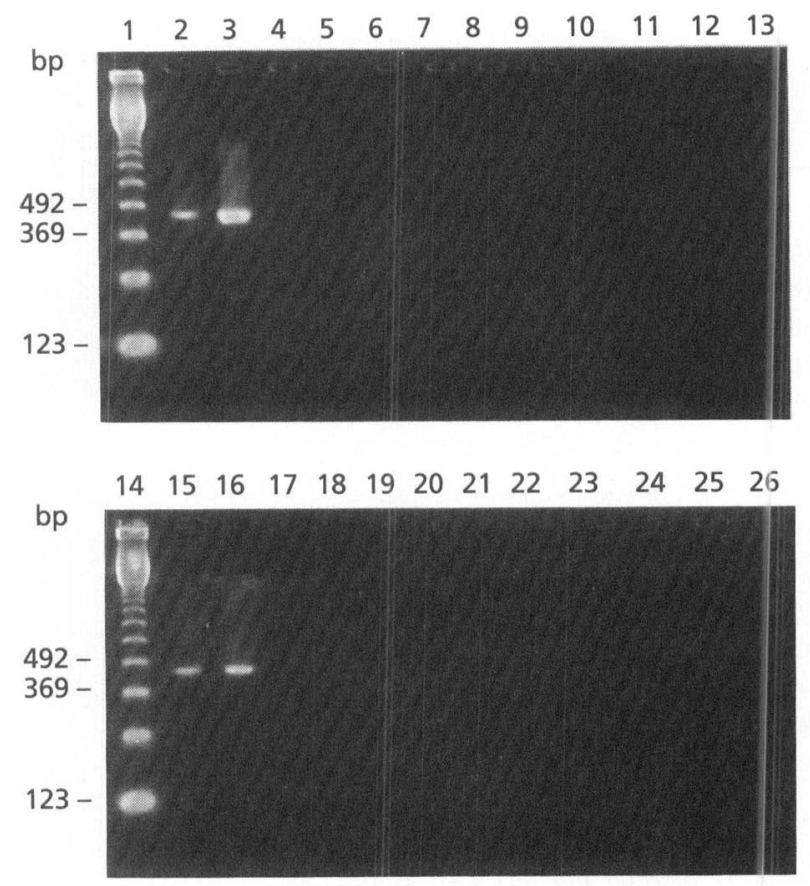

Fig. 4. Species-specific PCR identification of $H$. pullorum sp. nov. Genomic DNA (100 ng) of each $H$. pullorum strain or type strain (as listed in Table 1) was used as a substrate for PCR as described in Results. Tracks 1 and 14 contained the $123 \mathrm{bp}$ ladder (Gibco BRL); track 2, H. pullorum NCTC 12824; track 3, $H$. pullorum NCTC 12825; track 4, $H$. pylori; track 5, $H$. felis; track 6, H. mustelae; track $7, H$. acinonyx; track $8, H$. cinaedi; track 9 , $H$. fennelliae; track $10, H$. nemestrinae; track $11, H$. muridarum; track $12, H$. canis; track $13, ' F$. rappini'; track $15, H$. pullorum NCTC 12826; track $16, H$. pullorum NCTC 12827; track 17, C. hyointestinalis; track 18, C. fetus; track 19, C. helveticus; track $20, C$. upsaliensis; track 21, C. lari; track 22, C. coli; track 23, C. jejuni; track 24, C. sputorum; track 25, C. mucosalis; track 26, C. concisus.

will be a useful tool for screening clinical and veterinary samples for the presence of $H$. pullorum.

\section{Formal description of $\boldsymbol{H}$. pullorum sp. nov.}

(pullorum, from Latin noun pullus, chicken; after source of first isolation)

Gram-negative, non-spore-forming, gently curved, slender, rod-shaped cells, 3-4 $\mu \mathrm{m}$ in length. Cells carry an unsheathed monopolar flagellum. Typical darting motility. Microaerophilic. Grows microaerobically at $37{ }^{\circ} \mathrm{C}$ and $42{ }^{\circ} \mathrm{C}$. No growth under aerobic conditions or anaerobically [on TMAO medium]. Colonies pinpoint, non-pigmented, translucent and $\alpha$-haemolytic on $5 \%$ horse blood agar. Produces oxidase and reduces nitrate. Most strains $(14 / 16)$ produce catalase. No production of urease or alkaline phosphatase. No hydrolysis of indoxyl acetate. Resistant to cephalothin and cefoperazone. The $\mathrm{G}+\mathrm{C}$ content of the genomic DNA by thermal denaturation is $34-35 \mathrm{~mol} \%$. A $447 \mathrm{bp} \mathrm{PCR} \mathrm{amplicon} \mathrm{is} \mathrm{specifi-}$ cally generated using the primers $5^{\prime}$ ATG AAT GCT AGT TGT TGT CAG $3^{\prime}$ and $5^{\prime}$ GAT TGG CTC CAC
TTC ACA $3^{\prime}$ and an annealing temperature of $60^{\circ} \mathrm{C}$. Isolated from broiler and layer chickens, with or without vibrionic hepatitis. Also isolated from humans with gastrointestinal disease.

\section{Formal description of the type strain}

NCTC $12824^{\mathrm{T}}$ is the type strain of H. pullorum. This strain conforms to the description of the species given above. Its $\mathrm{G}+\mathrm{C}$ content is $34.5 \mathrm{~mol} \%$. It was isolated from a healthy broiler chicken in Switzerland. Three additional strains have been deposited with the National Collection of Type Cultures: NCTC 12825, NCTC 12826 and NCTC 12827 .

\section{ACKNOWLEDGEMENTS}

This work was initiated with the support of a British Council Grant to J. Stanley for British-Swiss scientific cooperation and supported in part by United States Public Health Service Grants (to Floyd Dewhirst) DE 08303 and DE 10374.

\section{REFERENCES}

Blaser, M. J. (1990). Helicobacter pylori and the pathogenesis of gastroduodenal inflammation. J Infect Dis 161, 626-633.

Blaser, J. (1993). Helicobacter pylori: microbiology of a 'slow' bacterial infection. Trends Microbiol 1, 255-260.

Boivin, M. F., Morris, V. L., Lee-Chan, E. C. M. \& Murray, R. G. E. (1985). Deoxyribonucleic acid relatedness between selected members of the genus Aquaspirillum by slot blot hybridization: Aquaspirillium serpens (Mueller 1786) Hylemon, Wells, Krieg and Jannasch 1973 emended to include Aquaspirillum bengal as a subjective synonym. Int J Syst Bacteriol 35, 512-517.

Burnens, A. P. \& Nicolet, J. (1992). Detection of Campylobacter upsaliensis in diarrheic dogs and cats, using a selective medium with cefoperazone. Am J Vet Res 53, 48-51.

Costas, M. (1992). Classification, identification and typing of bacteria by the analysis of their one-dimensional polyacrylamide gel electrophoresis protein patterns. In Advances in Electrophoresis, pp. 351-408. Edited by A. Chambrach, M. J. Dunn \& B. J. Radola. Weinheim: VCH Verlagsgesellschaft.

Dewhirst, F. E., Seymour, C., Fraser, G. J., Paster, B. J. \& Fox, J. G. (1994). Phylogeny of Helicobacter isolates from bird and swine feces and description of Helicobacter pametensis sp. nov. Int J Syst Bacteriol 44, 553-560.

Eaton, K. A., Dewhirst, F. E., Radin, M. J., Fox, J. G., Paster, B. J., Krakowka, S. \& Morgan, D. R. (1993). Helicobacter acinonyx sp. nov., isolated from cheetahs with gastritis. Int J Syst Bacteriol 43, 99-106.

Embley, T. M. (1991). The linear PCR reaction : a simple and robust method for sequencing amplified rRNA genes. Lett Appl Microbiol 13, 171-174.

Fox, J. G., Dewhirst, F. E., Tully, J. G., Paster, B. J., Yan, L., Taylor, N. S., Collins, M. J., Jr, Gorelick, P. L. \& Ward, J. M. (1994). Helicobacter hepaticus sp. nov., a microaerophilic bacterium isolated from the livers and intestinal mucosal scrapings from mice. J Clin Microbiol 32, 1238-1245.

Han, Y.-H., Smibert, R. M. \& Krieg, N. R. (1989). Occurrence of sheathed flagella in Campylobacter cinaedi and Campylobacter fennelliae. Int J Syst Bacteriol 39, 488-490.

Holmes, B., On, S. L. W., Costas, M. \& Ganner, M. (1992). Some new applications of probabilistic identification. In Proceedings of the 
Conference on Taxonomy and Automated Identification of Bacteria, pp. 6-8. Edited by J. Schindler. Prague: Czechoslovak Society for Microbiology.

Jukes, T. H. \& Cantor, C. R. (1969). Evolution of protein molecules. In Mammalian Protein Metabolism, pp. 21-132. Edited by H. N. Munro. New York: Academic Press.

Kusukawa, N., Uemori, T., Asada, K. \& Kato, I. (1990). Rapid and reliable protocol for direct sequencing of material amplified by the polymerase chain reaction. Biotechniques 9, 66-72.

Lee, A. S. G. \& McGee, J. O. (1989). The signal intensity on Southern blots developed by nonisotopic methods is linear with time and quantity of DNA. Nucleic Acids Res 17, 2364.

Lior, H. \& Patel, A. (1987). Improved toludine blue-DNA agar for detection of DNA hydrolysis by campylobacters. J Clin Microbiol 25, 2030-2031.

Mishu, B., Patton, C. M. \& Tauxe, R. V. (1993). Clinical and epidemiological features of non-jejuni, non-coli Campylobacter species. In Campylobacter jejuni: Current Status and Future Trends, pp. 31-41. Edited by I. Nachamkin, M. J. Blaser \& L. S. Tompkins. Washington, DC: American Society for Microbiology.

On, S. L. W. \& Holmes, B. (1991a). Effect of inoculum size on the phenotypic characterization of Campylobacter spp. J Clin Microbiol 29, 923-926.

On, S. L. W. \& Holmes, B. (1991b). Reproducibility of tolerance tests that are useful in the identification of campylobacteria. $J$ Clin Microbiol 29, 1785-1788.

On, S. L. W. \& Holmes, B. (1992). Assessment of enzyme detection tests useful in identification of campylobacteria. J Clin Microbiol 30, 746-749.

Owen, R. J. \& Pitcher, D. G. (1985). Chemical methods for estimating DNA base compositions and levels of DNA-DNA hybridization. In Chemical Methods in Bacterial Systematics, pp. 67-93. Edited by M. Goodfellow \& D. E. Minnikin. London: Academic Press.

Paster, B. J. \& Dewhirst, F. E. (1988). Phylogeny of Campy- lobacters, Wolinellas, Bacteroides gracilis, and Bacteroides ureolyticus by $16 \mathrm{~S}$ ribosomal ribonucleic acid sequencing. Int J Syst Bacteriol 38, $56-62$.

Saitou, N. \& Nei, M. (1987). The neighbour-joining method: a new method for reconstructing phylogenetic trees. Mol Biol Evol 4, 406-425.

Seymour, C., Lewis, R. J., Kim, M., Gagnon, D. F., Fox, J. G., Dewhirst, F. E. \& Paster, B. J. (1994). Isolation of Helicobacter strains from wild bird and swine feces. Appl Environ Microbiol 60, 1025-1028.

Stanley, J., Burnens, A. P., Linton, D., On, S. L. W., Costas, M. \& Owen, R. J. (1992). Campylobacter helveticus sp. nov., a new thermophilic species from domestic animals: characterization and cloning of a species-specific DNA probe. J Gen Microbiol 138, 2293-2303.

Stanley, J., Linton, D., Burnens, A. P., Dewhirst, F. E., Owen, R. J., Porter, A., On, S. L. W. \& Costas, M. (1993). Helicobacter canis sp. nov., a new species from dogs: an integrated study of phenotype and genotype. J Gen Microbiol 139, 2495-2504.

Stern, N. J. (1993). Reservoirs for Campylobacter jejuni and approaches for intervention in poultry. In Campylobacter jejuni: Current Status and Future Trends, pp. 49-60. Edited by I. Nachamkin, M. J. Blaser \& L. S. Tompkins. Washington, DC: American Society for Microbiology.

Totten, P. A., Fennell, C. L., Tenover, F. C., Wezenberg, J. M., Perine, P. L., Stamm, W. E. \& Holmes, K. K. (1985). Campylobacter cinaedi (sp. nov.) and Campylobacter fennelliae (sp. nov.): two new Campylobacter species associated with enteric disease in homosexual men. $J$ Infect Dis 151, 131-139.

Vandamme, P., Falsen, E., Rossau, R., Hoste, B., Segers, P., Tytgat, R. \& De Ley, J. (1991). Revision of Campylobacter, Helicobacter, and Wolinella taxonomy: emendation of generic descriptions and proposal of Arcobacter gen. nov. Int J Syst Bacteriol 41, 88-103.

Received 9 May 1994; revised 1 August 1994; accepted 3 August 1994. 